\title{
Evaluation of Conduit in Total Arterial Revascularization using Optical Coherence Tomography (OCT) - A Case Report and Literature Review
}

\author{
Angelo Citarella ${ }^{1}$, Abdul Sheikh Sheikh ${ }^{1}$, Saqib Chowdhary ${ }^{1}$, and Nnamdi Nwaejike ${ }^{1}$ \\ ${ }^{1}$ Wythenshawe Hospital
}

April 29, 2020

\begin{abstract}
Coronary artery bypass grafting $(\mathrm{CABG})$ remains the preferred revascularization modality for the elective treatment of patients with complex three vessel coronary artery disease. There is evidence that the use of additional arterial grafts such as right internal mammary artery (RIMA) and Radial Artery (RA); are associated with improved long-term patency. Stenosis of arterial grafts can occur in some patients postoperatively. We describe the first use of OCT in the evaluation of suspected conduit stenosis in Total Arterial Revascularisation.
\end{abstract}

\section{Introduction}

Coronary artery bypass grafting (CABG) remains the preferred revascularization modality for the elective treatment of patients with complex three vessel coronary artery disease. The left internal mammary artery (LIMA) is routinely used for revascularization of the left anterior descending artery. However, the choice of conduit used in addition to the LIMA can be contentious. There is evidence that the use of additional arterial grafts such as right internal mammary artery (RIMA) and Radial Artery (RA); are associated with improved long-term patency. LIMA grafting to the left anterior descending (LAD) artery and use of a radial artery instead of the saphenous vein are Class I recommendations in the 2018 ESC/EACTS guidelines on myocardial revascularization. The use of bilateral IMA conduits is a Class IIa recommendation. Stenosis of arterial grafts can occur in some patients postoperatively. However, no reports have described an evaluation of conduit using optical coherence tomography (OCT) to ascertain the mechanism of stenosis. We describe the use of OCT in the evaluation of suspected conduit stenosis in Total Arterial Revascularisation.

\section{Case Summary}

A 63 years old lady who was admitted with acute coronary syndrome. She was referred for coronary artery bypass surgery. Risk factors included previous PCI and stents to LAD and right coronary artery (RCA); arteriopathy with peripheral vascular disease treated with stents in both iliac arteries; persistent intermittent claudication; pulmonary emphysema and current smoker. Left heart catheterization (LHC) showed severe triple vessel disease with preserved left ventricular systolic function. She underwent a triple vessel coronary artery bypass surgery by Total Arterial Revascularization using cardio-pulmonary bypass. The LIMA and the RIMA were harvested in skeletonized fashion and a T-anastomosis (Tector) was performed. LIMA was grafted to LAD and RIMA sequentially to distal Circumflex (LCx) and posterior descending artery (PDA). (Figure 1)

Temporary atrial pacing wires were placed and the patient was transferred to cardiothoracic intensive therapy unit in haemodynamically stable condition. The patient had an asystolic cardiac arrest with no pacemaker capture 2 hours later. There were no ECG changes suggestive of myocardial ischaemia leading to the event. 
Cardiac surgery Advanced Life Support (CALS) was initiated and the patient was stabilised on central VenoArterial-Extra Corporeal Membrane Oxygenation (VA-ECMO). Bedside transesophageal echocardiogram (TOE) showed a global severe depressed left ventricular function which was confirmed on heart inspection when the chest was reopened. When stabilized, emergency LHC was performed and it showed patent LIMA to LAD with good run off distally. RIMA showed a stenosis just distal to the Y-anastomosis. There was good run off into PDA and obtuse marginal (OM) artery. (Figure 2) We did OCT to evaluate the mechanism of stenosis. A large intramural haematoma was shown to be compressing the lumen. There was no evidence of intimal dissection (Figure 3). Patient was managed conservatively as there was no flow limitation in the grafts.

\section{Discussion}

Likely mechanisms of intra-mural haematoma in this case include surgical trauma perioperatively or trauma/bruising to the graft from cardiac massage during the CALS protocol. We routinely use skeletonized mammary arteries for CABG. Intra-operative surgical trauma seemed unlikely as there was no visible evidence of damage to the conduit during the operation. An evolving haematoma would more likely present as progressive regional ischaemia on ECG with segmental wall motion abnormality on echocardiogram. There were no ischaemic changes on ECG post-operatively. This patient had no regional wall motion abnormality on postcardiopulmonary bypass transoesophageal echocardiogram (TOE). Post-resuscitation TOE showed global LV impairment after stabilisation on VA-ECMO. It could be that the cardiac arrest $2 \mathrm{hrs}$ after surgery was unrelated to the intramural haematoma and the haematoma was as a result of cardiac massage during CPR.

A much more common cause of early graft failure (failure within 1 month of surgery) is technical failure or thrombosis which is usually at the site of the anastomosis. LHC and OCT showed that all anastomoses were intact with good flow and no sign of intra-lumnal thrombosis.

OCT is a catheter based invasive imaging system which allows for high-resolution imaging of intra-luminal and trans-mural coronary structures thus overcoming many of the limitations of angiography. Using light rather than ultrasound, OCT produces high-resolution in-vivo images of coronary arteries and deployed stents.

Accumulating data support a clinical role for OCT in a multitude of clinical scenarios, including assessing the natural history of atherosclerosis and modulating effects of therapies, mechanisms of acute coronary syndromes, mechanistic insights into the effects of novel interventional devices, and optimization of percutaneous coronary intervention. We have found no previous reports of the use of OCT to evaluate conduit post-CABG. Potential risks from the procedure include that it can cause intimal dissection in fresh conduit.

Should OCT be used to routinely evaluate all conduit post-op? We can advocate its usage when the LHC findings are unclear.

OCT to complement LHC can be a valuable tool to evaluate conduit post CABG. The absence of proximal aortic anastomoses in total arterial re-vascularization reduces the risk of graft disruption when early postoperative LHC/OCT is indicated. 


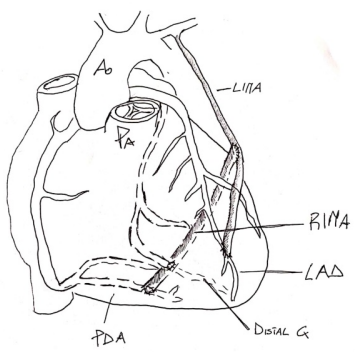

Fig.1: LIMA to LAD, RIMA (y Graft to LIMA) to Distal Cx and PDA. 


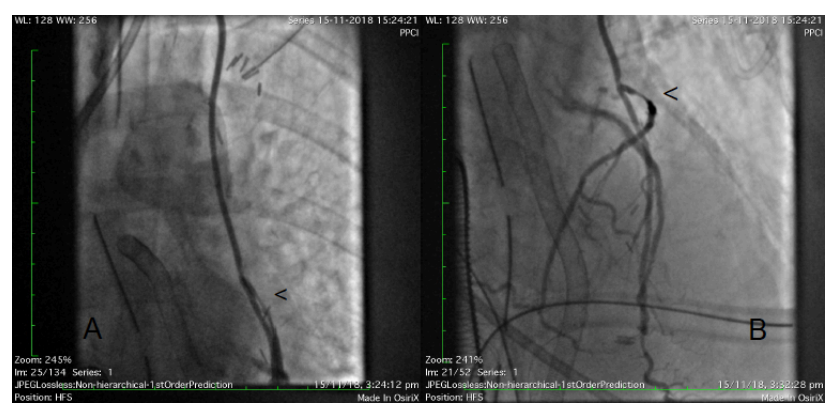

Figure 2: LIMA LHC showing (A) patent LIMA with good distal run off in LAD and severe stenosis at the proximal segment of free RIMA T graft \& (B) severe stenosis at the proximal segment of free RIMA T graft and good run off into PDA and $\mathrm{OM}$. 


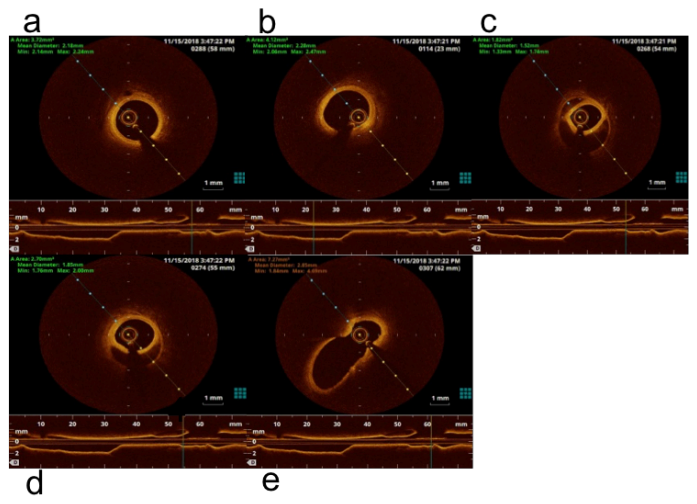

Figure 3: OCT pull back from free RIMA graft back into LIMA showing (a) widely open lumen and normal three layers of vessel wall in mid segment of RIMA, (b) start of intramural haematoma in proximal segment of RIMA, (c) segment of severe

luminal stenosis due to external compression, (d) segment of intramural

haematoma compressing lumen, (e) widely open anastomosis of RIMA into LIMA. 